\title{
Adequate feeding and the usefulness of the respiratory quotient in critically ill children
}

\author{
Jessie M. Hulst, M.D. ${ }^{\mathrm{a}, \mathrm{b}}$, Johannes B. van Goudoever, M.D., Ph.D ${ }^{\mathrm{b}}$, \\ Luc J. Zimmermann, M.D., Ph.D ${ }^{b}$, Wim C. Hop, M.Sc, Ph.D ${ }^{c}$, Hans A. Büller, M.D., Ph.D ${ }^{b}$, \\ Dick Tibboel, M.D., Ph.D ${ }^{\mathrm{a}}$, Koen F. M. Joosten, M.D., Ph.D ${ }^{\mathrm{b}, *}$ \\ a Department of Pediatric Surgery, Erasmus MC, Sophia Children's Hospital, Rotterdam, The Netherlands \\ ' Department of Pediatrics, Erasmus MC, Sophia Children's Hospital, Rotterdam, The Netherlands \\ ${ }^{\mathrm{c}}$ Department of Biostatistics and Epidemiology, Erasmus MC, Rotterdam, The Netherlands
}

Manuscript received October 24, 2003; accepted May 26, 2004.

\begin{abstract}
Objective: We determined incidences of underfeeding and overfeeding in children who were admitted to a multidisciplinary tertiary pediatric intensive care and evaluated the usefulness of the respiratory quotient (RQ) obtained from indirect calorimetry to assess feeding adequacy.

Methods: Children $18 \mathrm{y}$ and younger who fulfilled the criteria for indirect calorimetry entered our prospective, observational study and were studied until day 14. Actual energy intake was recorded, compared with required energy intake (measured energy expenditure plus 10\%), and classified as underfeeding ( $<90 \%$ of required), adequate feeding ( $90 \%$ to $110 \%$ of required), or overfeeding ( $>110 \%$ of required). We also evaluated the adequacy of a measured RQ lower than 0.85 to identify underfeeding, and an RQ higher than 1.0 to identify overfeeding.

Results: Ninety-eight children underwent 195 calorimetric measurements. Underfeeding, adequate feeding, and overfeeding occurred on $21 \%, 10 \%$, and $69 \%$ of days, respectively. An RQ lower than 0.85 to identify underfeeding showed low sensitivity $(63 \%)$, high specificity (89\%), and high negative predictive value $(90 \%)$. An RQ higher than 1.0 to indicate overfeeding showed poor sensitivity (21\%), but a high specificity (97\%) and a high positive predictive value (93\%). Food composition, notably high-carbohydrate intake, was responsible for an RQ exceeding 1.0 in the overfed group.

Conclusion: Children admitted to the intensive care unit receive adequate feeding on only $10 \%$ of measurement days during the first $2 \mathrm{wk}$ of admission. The usefulness of RQ to monitor feeding adequacy is limited to identifying (carbohydrate) overfeeding and excluding underfeeding. (c) 2005 Elsevier Inc. All rights reserved.
\end{abstract}

\section{Introduction}

Nutritional support is essential in the care of critically ill children because inadequate feeding can increase morbidity and mortality rates [1]. Critically ill children who receive adequate nutritional support have shown significant improvement in physiologic stability and outcome $[2,3]$. Fur-

This study was supported in part by Nutricia Nederland BV, Zoetermeer, The Netherlands.

* Corresponding author. Tel.: +3110-4637029; fax: +3110-4636796.

E-mail address: k.joosten@erasmusmc.nl (K.F.M. Joosten). ther, the goal of supporting critically ill children is not only to restore a normal functioning level but also to meet the requirements for growth and development. Thus clinicians in the pediatric intensive care unit (ICU) are challenged to provide adequate energy for optimal tissue synthesis and immune function and avoid complications of overfeeding.

The major problem in clinical practice is to define nutritional requirements for critically ill children because demands range widely, with altered metabolic states determined by a child's age, state of health, and nutritional status. Moreover, metabolic responses may greatly vary, depending on the nature of the injury and the variability of the 
individual response to the same type of injury [4,5]. Because standard feeding protocols do not provide for interindividual differences in energy requirements and because the available prediction equations are inadequate for calculating energy needs in critically ill children, these children are more vulnerable to complications that arise from underfeeding or overfeeding.

Therefore, it is important to monitor whether children admitted to an ICU are adequately fed. Indirect calorimetry provides a method that can help in this matter. In studies in adults, feeding adequacy was determined by the ratio of energy intake (EI) to measured energy expenditure (MEE) plus $10 \%$ for activity [6]. A second parameter derived from indirect calorimetry is the respiratory quotient $(\mathrm{RQ})$, which is the ratio of carbon dioxide produced to oxygen consumed. The role of the RQ as a marker of substrate use is controversial in critical illness because the body's ability to use nutrient substrates may be altered. Moreover, the clinical usefulness of the RQ as an indicator of underfeeding or overfeeding has been found to be limited in adult critically ill patients due to its low sensitivity and specificity [7]. McClave et al. [7] concluded that its clinical usefulness is restricted to a marker of test validity and a marker of respiratory tolerance of the nutritional support regimen. Previous studies among critically ill children using indirect calorimetry have not specified criteria for adequate feeding; the only criterion specified to define overfeeding is a measured RQ higher than $1.0[4,8]$.

We developed this study with two objectives: 1) to determine incidences of underfeeding and overfeeding in a heterogeneous cohort of children who had been admitted to the pediatric ICU of our level III children's hospital, based on the criterion used in adults, and 2) to evaluate whether the RQ could be used to monitor whether these children were adequately fed.

\section{Materials and methods}

\section{Subjects}

Over a 1-y period (2001) children 18 y and younger who had been admitted to our level III multidisciplinary pediatric or surgical ICU with an expected stay of at least $48 \mathrm{~h}$ were studied repeatedly by indirect calorimetry during the first $14 \mathrm{~d}$ of admission. The institutional review board of the Erasmus MC (Rotterdam, The Netherlands) approved the study protocol, and written (parental) informed consent was obtained before subjects entered the study. Exclusion criteria for this study were withholding or withdrawing of treatment and inclusion into a nutritional intervention study. In addition, children were excluded if they did not fulfill the inclusion criteria for indirect calorimetric measurements. The following inclusion criteria had to be satisfied for indirect calorimetry in ventilated children: 1) mechanical ventilation with a Servo Ventilator 300 (Siemens-Elema, Solna,
Sweden); 2) inspired oxygen fraction less than 60\%; 3) tube leakage less than $10 \%$ (considered not to significantly affect the measurements; determined by comparing inspired with expired tidal volumes measured by the ventilator, assuming an absence of air leaks in the circuit between patient and ventilator) [9]; and 4) a hemodynamically stable condition (within two standard deviations of age-related values for blood pressure and heart rate) [10]. Non-ventilated children who received supplementary oxygen were excluded from the measurements because of the technical issues involved.

Clinical data collected included age, sex, weight, primary diagnosis, surgical status, days on mechanical ventilation, length of stay in the ICU, route of nutritional support, and EI. Severity of illness on admission was assessed by the Pediatric Risk of Mortality score [11]. Weight was measured on each measurement day according to the methods described by Gerver and De Bruin [12].

\section{Energy expenditure}

Indirect calorimetric measurements were started as soon as technically possible after admission to the ICU and continued on each subsequent day in respiratory or canopy mode, depending on the child's need for mechanical ventilation. Oxygen consumption, carbon dioxide production, and RQ standardized for temperature, barometric pressure, and humidity were measured with the Deltatrac II MBM200 (Datex Division Instrumentarium, Helsinki, Finland) metabolic monitor, and MEE was calculated with the modified Weir's formula [13]. The properties of the Deltatrac metabolic monitor have been described previously [14]. Before each study, the calorimeter was calibrated with a reference gas mixture $\left(95 \% \mathrm{O}_{2}, 5 \% \mathrm{CO}_{2}\right.$; Datex Division Instrumentarium). Data for the first $5 \mathrm{~min}$ were routinely discarded to exclude artifacts that arose during connection of the calorimeter to the patient. Measurements in the ventilated children lasted at least $2 \mathrm{~h}$. Measurements in nonventilated children lasted at least $20 \mathrm{~min}$. Placed in an airtight transparent plastic canopy, these children were sleeping or quietly resting in supine position and had not been exposed to diagnostic or therapeutic procedures in the previous $30 \mathrm{~min}$. Flow range and canopy size were in conformity with the manufacturer's instructions. Measurement results in canopy mode were used in the analysis only if steady states in $\mathrm{O}_{2}$ and $\mathrm{CO}_{2}$ concentrations had been obtained. Steady-state criteria were defined as a coefficient of variation of oxygen consumption and carbon dioxide production levels no greater than $10 \%$ for at least $15 \mathrm{~min}$.

\section{Energy intake}

Children were enterally and/or parenterally fed on the guidance of the current feeding protocol and the judgment of the physician clinically responsible for a particular child, independent of the study. During the first 12 to $24 \mathrm{~h}$, a glucose infusion was given. Fluid and electrolyte intakes 
were adjusted to individual requirements. For neonates, the amount of enteral or parenteral feeding was increased in accordance with increasing fluid intake until the amount was 150 to $180 \mathrm{~mL} \cdot \mathrm{kg}^{-1} \cdot \mathrm{d}^{-1}$ at day 7 . If enteral feeding could not be started on the second day, total parenteral feeding was started within 24 to $48 \mathrm{~h}$ after admission by peripheral infusion or by a central venous line (Intralipid 20\%, Pharmacia Upjohn, Woerden, The Netherlands; Primene $10 \%$ [body weight $\leq 5 \mathrm{~kg}$ ), Clintec Baxter, Mississauga, Canada; Aminovenös N-paed 10\% [body weight $>5 \mathrm{~kg}$ ], Fresenius, Hertogenbosch, The Netherlands). On the first day of parenteral feeding, amounts of protein and fat were $50 \%$ of the desired intake according to hospital practice ( 1 to $2.4 \mathrm{~g} \cdot \mathrm{kg}^{-1} \cdot \mathrm{d}^{-1}$ and 2 to $4 \mathrm{~g} \cdot \mathrm{kg}^{-1} \cdot \mathrm{d}^{-1}$, respectively, depending on age). Enteral feeding was started as soon as possible in all children, continuously or intermittently, through a nasogastric tube (drip or bolus) or nasoduodenal tube (drip), consisting of human milk or standard formula according to the parents' preference (Nenatal for preterm neonates, Nutrilon Premium for children younger than 1 mo, Nutrison Pediatric Standard for children ages 1 mo to $1 \mathrm{y}$, and Nutrison Multi Fiber for children older than 1 y; Nutricia, Zoetermeer, The Netherlands).

Actual total daily EI (kcal/d) was determined by patientintake records on the day of calorimetry, after which EI/ MEE could be calculated. We corrected for additional protein calories from sources such as plasma and/or albumin infusions and for extra carbohydrate calories from medication solutions. EIs on the day of admission and the day of discharge were extrapolated to a 24-h intake. To determine the composition of feeding, an RQ of administered macronutrients was obtained from the modified Lusk table after determining the ratio of carbohydrate to fat for the total non-protein calories of the nutrients [15].

Energy requirements $(\mathrm{kcal} / \mathrm{d})$ were defined as MEE plus $10 \%$ for patient activity related to nursing care ( $110 \%$ of MEE) as used by McClave et al. in critically ill adults [6]. As a consequence, the degree of feeding was defined as $\mathrm{EI} /(\mathrm{MEE}+10 \%)$. Underfeeding was defined as a ratio lower than $90 \%$, adequate feeding as a ratio of $90 \%$ to $110 \%$, and overfeeding as a ratio higher than $110 \%$. The adequacy of feeding as classified by EI/(MEE $+10 \%$ ) was compared with the classification based on the measured RQ [7]. With the measured RQ, underfeeding was defined as an RQ lower than 0.85 and overfeeding as as RQ higher than 1.0. We calculated the specificity, sensitivity, positive predictive value, and negative predictive value of measured RQ values lower than 0.85 and higher than 1.0 to correctly identify underfeeding and overfeeding, respectively.

\section{Statistical analysis}

Statistical analyses were performed with SPSS 11.0 for Windows (SPSS, Inc., Chicago, IL, USA). Results are expressed as mean \pm standard error of the mean or as median
Table 1

Patient characteristics and diagnostic information*

\begin{tabular}{ll}
\hline & $\begin{array}{l}\text { Total group } \\
(n=98)\end{array}$ \\
\hline Male/female $^{\dagger}$ & $50 / 48$ \\
Age at admission & $14.5 \mathrm{~d}(0 \mathrm{~d}$ to $15.2 \mathrm{y})$ \\
Length of stay (d) & $7(2-314)$ \\
Days on mechanical ventilation & $3(0-47)$ \\
PRISM score at admission & $12(0-38)$ \\
Diagnostic groups & \\
Congenital anomalies $^{\ddagger}$ & 36 \\
Postnatal problems & \\
$\quad$ Postoperative monitoring & 14 \\
Sepsis or meningitis & 16 \\
$\quad$ Respiratory illness & 10 \\
Other & 15 \\
Surgical/non-surgical & \\
\end{tabular}

PRISM, Pediatric Risk of Mortality

* Values expressed as median (range), except when indicated otherwise.

${ }^{\dagger}$ Expressed as numbers.

* Predominant diagnoses.

$\S$ Includes asphyxia, meconium aspiration, and infection.

"Includes pneumonia and rs-bronchiolitis.

and range. To allow optimally for the variable number of measurement days and take into account between- and within-patient variations, repeated measures analysis of variance (PROC MIXED, SAS, Cary, NC, USA) was used to compare mean values and examine relations between various parameters. This analysis was used to examine relations between RQ and various clinical factors (day after admission, EI/MEE, RQ of administered macronutrients, carbohydrate intake $\left[\mathrm{mg} \cdot \mathrm{kg}^{-1} \cdot \mathrm{min}^{-1}\right]$, fat intake $\left[\mathrm{g} \cdot \mathrm{kg}^{-1}\right.$. $\mathrm{d}^{-1}$ ], route of feeding [enteral, parenteral, or combination], age, and Pediatric Risk of Mortality scores). Two-tailed $P$ values lower than 0.05 were considered statistically significant.

\section{Results}

\section{Clinical characteristics}

One hundred twelve children were eligible for this study. However, 14 children were excluded from analysis because of invalid results (e.g., did not reach steady state) or incomplete nutritional data. Thus 98 children comprised the final study group, and their clinical characteristics at admission are presented in Table 1.

A total of 195 measurements, 83 in canopy mode and 112 in respiratory mode, were performed. In 49 children $(50 \%)$, more than one valid indirect calorimetric measurement could be obtained during the first $14 \mathrm{~d}$ of admission. Measurement characteristics are listed in Table 2. Because additional protein and carbohydrate losses from wounds or drained pleural and peritoneal effusions were not significant on the measurement days, these were not adjusted for in the EI calculations. 
Table 2

Characteristics of indirect calorimetric measurements and days on which the measurements were performed*

\begin{tabular}{lc}
\hline & $\begin{array}{c}\text { All measurements } \\
(n=195)\end{array}$ \\
\hline Measurement mode: canopy/respiratory & $83 / 112(43 / 57)$ \\
Day of measurement & $2(0-14)$ \\
Respiratory mode & $4(0-14)$ \\
Canopy mode & $4(0-14)$ \\
Total & $2(0-14)$ \\
Day of first measurement & $2(1-7)$ \\
No. of measurements/child & \\
Duration of measurements (min) & $119(60-794)$ \\
Respiratory mode & $24(15-47)$ \\
Canopy mode & $112(57)$ \\
Medication & $15(8)$ \\
Sedatives & $8(4)$ \\
Cardiotonics & \\
Muscle relaxants & $1(0-10)$ \\
Ventilator characteristics & $79 / 32 / 1(71 / 28 / 1)$ \\
Tube leakage $\%)$ & \\
PRVC/VS/PS & $38(19)^{\dagger}$ \\
Nutritional support & $80(41)$ \\
Parenteral nutrition & $77(40)$ \\
Parenteral plus enteral nutrition & \\
Enteral nutrition &
\end{tabular}

PRVC, pressure-regulated volume control; PS, pressure support; VS, volume support.

* Values are expressed as median (range) or numbers (\%).

${ }^{\dagger}$ Five children received a glucose infusion exclusively.

For the total study population, the mean EI/MEE increased with increasing time in the ICU (Fig. 1), with $8.1 \pm$ $1.2 \%$ per day $(P<0.001)$. Altogether, EI was lower than MEE on 21\% (41 of 195) of measurement days and higher than MEE on $79 \%$ of days.

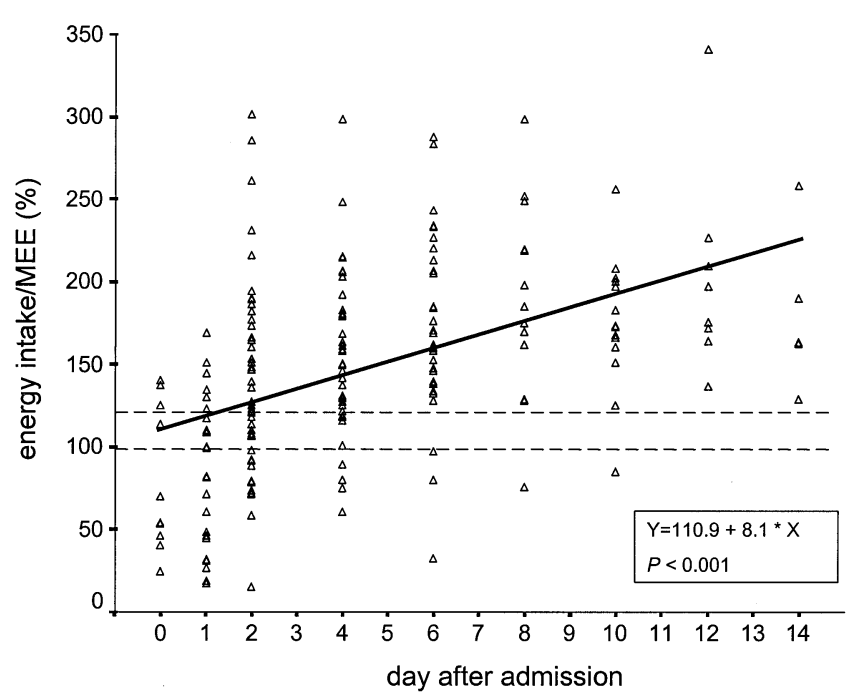

Fig. 1. Relation between ratio of energy intake to MEE and day after admission. Data points $(n=195)$ with fitted regression line were generated by repeated measures analysis of variance. The area between the dashed lines indicates the reference range for adequate feeding, defined as $90 \%$ to $110 \%$ of the ratio of energy intake to MEE $+10 \%$. MEE, measured energy expenditure.

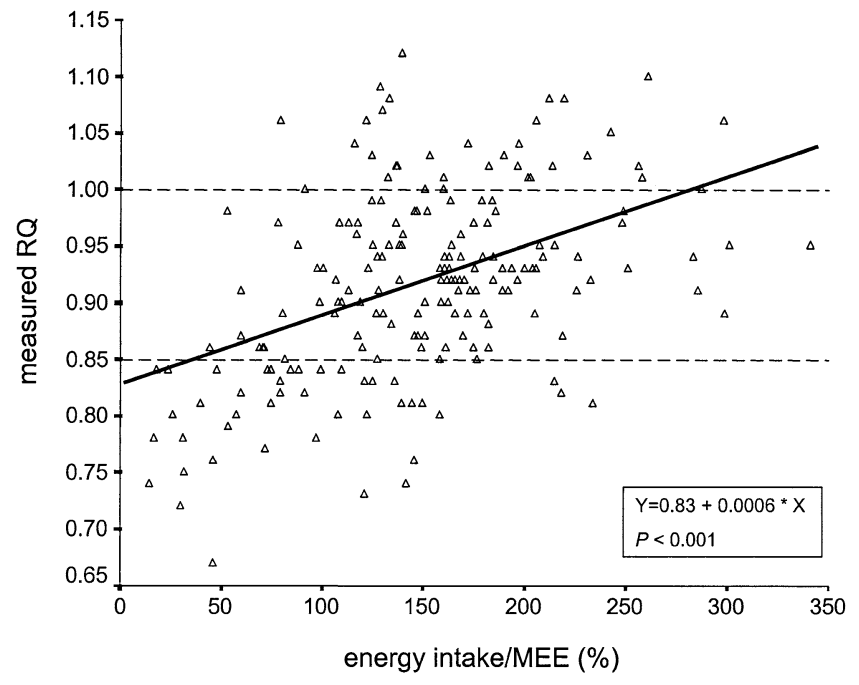

Fig. 2. Relation between ratio of energy intake to MEE and measured RQ. Data points $(n=195)$ with fitted regression line were generated by repeated measures analysis of variance. The area between the dashed lines indicates the reference range for adequate feeding according to measured RQ. MEE, measured energy expenditure; RQ, respiratory quotient.

The positive relation between EI/MEE and measured RQ was highly significant, as shown in Fig. $2(P<0.001)$. Three other clinical factors were independently related to RQ. In addition to EI/MEE, positive relations were found with day after admission $(P=0.0001)$, EI/MEE $(P<$ $0.001)$, and carbohydrate intake $(P=0.003)$. A negative association was found for fat intake $(P<0.001)$. Route of feeding, age, and Pediatric Risk of Mortality score at admission were not significantly related to RQ.

\section{Sensitivity, specificity, and positive and negative predictive values of $R Q$}

Underfeeding, adequate feeding, and overfeeding were noted on $21 \%, 10 \%$, and $69 \%$ of measurement days, respectively. Table 3 presents the relation between the classification based on measured RQ and the degree of feeding by all measurements $(n=195)$. Using a measured RQ lower than 0.85 to identify underfeeding had a low sensitivity of $63 \%$ and a specificity of $89 \%$. The positive predictive value was $60 \%$, and the negative predictive value was $90 \%$. Using a

Table 3

Measured RQ in relation to degree of feeding*

\begin{tabular}{lclc}
\hline Degree of feeding $(n=195)$ & \multicolumn{3}{l}{ Measured RQ } \\
\cline { 2 - 4 } & $<0.85$ & $0.85-1.0$ & $>1.0$ \\
\hline Underfeeding $(n=40)$ & $25(63)$ & $14(35)$ & $1(2)$ \\
Adequate feeding $(n=20)$ & $5(25)$ & $14(70)$ & $1(5)$ \\
Overfeeding $(n=135)$ & $12(9)$ & $95(70)$ & $28(21)$
\end{tabular}

$\mathrm{RQ}$, respiratory quotient

* Data represent numbers of measurement days (\%). 
measured RQ higher than 1.0 to identify overfeeding had a poor sensitivity of $21 \%$ and a specificity of $97 \%$. The positive predictive value was $93 \%$, and the negative predictive value was $35 \%$.

Similar values for sensitivity, specificity, positive predictive value, and negative predictive value were found when measurements were broken down according to admission day.

\section{Discrepancies between criteria}

Comparison of the days with underfeeding $(n=40)$ with and without a measured RQ lower than 0.85 showed that mean carbohydrate intake was significantly lower on days with an RQ lower than 0.85. Comparison of days with overfeeding ( $n=135)$ with and without an RQ higher than 1 showed that EI/MEE did not significantly differ, whereas significantly higher mean carbohydrate intakes $(P=0.007)$ and significantly lower mean fat intakes $(P=0.018)$ were registered for those with an RQ higher than 1.0. Only on 2 of the 30 measurement days with an RQ higher than 1.0, no overfeeding occurred; a high carbohydrate intake (3.2 and $6.0 \mathrm{mg} \cdot \mathrm{kg}^{-1} \cdot \mathrm{min}^{-1}$ ) could not explain the finding of an RQ higher than 1.0 in these two measurements.

\section{Discussion}

In this study we analyzed relations between MEE, actual EI, and measured RQ in a mixed cohort of children who had been admitted to our ICU. On $21 \%$ and $79 \%$ of measurement days, EI was below and above MEE, respectively. By applying a classification used for adults [6,7], children would have received adequate nutrition on only $10 \%$ of days, whereas children would have been underfed and overfed on $21 \%$ and $69 \%$ of days, respectively.

The large proportion of overfeeding noted in this study is remarkable. The cutoff value of $110 \%$ for EI/(MEE $+10 \%$ ) to indicate overfeeding (which corresponds to $121 \%$ of MEE), as used for adults by McClave et al., is beyond discussion for critically ill children in the acute phase of metabolic stress [8]. However, their criterion for overfeeding is arguable in critically ill children who have resumed somatic growth. As opposed to critically ill adults, the feeding regimen for children must also take into account the energy for this growth $[16,17]$. Because growth-related energy can be measured only partly with indirect calorimetry, the limits for adequate and overfeeding will depend on the age of the child. Further, it is not exactly known when the acute phase turns into the recovery phase. A recent study in critically ill children using endocrine parameters showed that return to anabolism may occur within the first few days after admission [18]. Another postoperative study that evaluated hormone profiles in children of different age groups reported that the metabolic stress response is significantly shorter in children than in adults [19]. Previous studies in critically ill children on mechanical ventilation and children with burns have suggested that $20 \%$ to $50 \%$ should be added to the MEE to reach adequate nutrition and achieve a positive nitrogen balance [20-22]. In recovering young infants, this amount of energy arguably might be even higher in case of catch-up growth $[16,17]$.

Currently there is no uniform standard for defining underfeeding and overfeeding in critically ill children in relation to possible adverse effects such as protein-energy malnutrition for underfeeding and respiratory problems or liver steatosis for overfeeding $[8,23]$. Therefore, there is need for a tool to assess adverse consequences of inadequate feeding and to indicate when nutritional support should be adjusted for each child. We wondered whether the measured RQ might be such a tool in critically ill children. In theory, the overall measured RQ should reflect the used proportions of fat, protein, and carbohydrates because each of these substrates has its unique RQ. Therefore, energy overfeeding or carbohydrate intake in excess of oxidation capacity with resulting lipogenesis would increase the RQ to a level above 1.0. The use of endogenous fat stores during underfeeding to meet caloric requirements would decrease the RQ below 0.85 . Used in this way, the measured RQ would act as an alert to the adverse consequences of inadequate feeding and theoretically might be used to evaluate nutritional adequacy.

With regard to all measurement days, an increase in EI/MEE resulted in a significant increase in measured RQ. This might argue for the usefulness of the RQ as a tool to assess feeding adequacy. However, when evaluating the usefulness of a single measured RQ in a specific range to classify the degree of feeding, we found poor sensitivity (63\% for underfeeding and $21 \%$ for overfeeding), which is in accordance with findings by McClave et al. in adults [7]. This means that the RQ is not reliably reflected in the same way in all children in response to overfeeding and underfeeding and, hence, that factors other than EI/MEE must be important. These issues limit the overall value of the RQ for monitoring the adequacy of nutritional support.

As opposed to low values for sensitivity, high values were found for specificity to identify overfeeding (97\%) and underfeeding (89\%) when using RQ values higher than 1.0 and lower than 0.85 , respectively. This means that only a few measurement days were indicated as days with underfeeding and overfeeding by RQ when there was actually no underfeeding or overfeeding (false-positive results). The usefulness of the RQ to correctly classify feeding adequacy in practice could be more directly assessed from the positive and negative predictive values. Considering the high negative predictive value (90\%) of an RQ lower than 0.85 , we may conclude that the finding of an RQ higher than 0.85 with reasonable certainty can exclude underfeeding. Further, because of the very high chance of correctly classifying a child as overfed when finding an RQ higher than 1.0 (positive predictive value $=93 \%$ ), a measured RQ higher than 1.0 can be useful in clinical practice. The usefulness of the RQ to recognize (carbohydrate) overfeeding has been 
demonstrated in other studies in critically ill children and adults $[4,8,24]$.

We found that levels of carbohydrate intake and EI/MEE were independently related to RQ. In addition, on those days with overfeeding with an RQ higher than 1.0, we found that a significantly different composition of feeding (higher carbohydrate intake, lower fat intake) was administered than on the days with overfeeding with an RQ lower than 1.0. In clinical practice this means that, when an RQ higher than 1.0 is found, carbohydrate intake and/or total EI has to be adjusted.

Our study has some limitations. First, measurements were not performed in a sequential manner in all children and possibly in ventilatory and non-ventilatory modes under different nutritional support regimens. Theoretically it would have been better to measure all patients systematically over the first $14 \mathrm{~d}$ in one mode and on a standard feeding regimen. It was our intention to measure every other day, but due to practical limitations this was not always possible. Second, looking into reasons for feeding days to fall within an RQ range that did not correspond to the feeding degree (discrepancies between criteria), we focused on composition of feeding. However, numerous factors, related and unrelated to feeding, can alter the value of a measured RQ in critically ill patients, e.g., hyperventilation, acidosis, effects of cardiotonic agents and neuromuscular blocking, and an individual response to a given substrate load, injury, or disease [25]. In addition, the ongoing use of endogenous fuels (protein catabolism, lipolysis), which were observed even when supplying adequate amounts of exogenous substrates, can result in discrepancies between measured RQ and the amount of energy provided. Third, our study group consisted of a heterogeneous group of children with different ages, diagnoses, and illness severity scores. In addition, measurements were performed under different nutritional support regimens. We deliberately opted for this epidemiologic approach, including almost all admitted patients, to investigate the metabolic monitor as a clinical tool in a pediatric population representative of any pediatric ICU. This study is embedded in a study that aims to provide guidelines for nutritional and metabolic monitoring in daily clinical practice, taking into account the patient mix usually seen in pediatric ICUs. We are aware that this inhomogeneity has various drawbacks and makes it difficult to interpret our results for specific groups of critically ill children such as septic or postoperative patients. We considered several possible influencing factors and found that route of feeding, age, and illness severity score at admission did not significantly affect the RQ.

In conclusion, when applying criteria used in critically ill adults, we found that critically ill children were overfed on most measurement days. Nevertheless, only on $20 \%$ of these days did there appear to be a chance to develop adverse consequences of overfeeding (RQ $>1.0$ ). In the pediatric ICU setting, the usefulness of the RQ to monitor feeding adequacy and to adjust nutritional support is limited due to poor sensitivity and large interindividual variability. However, the extreme values of RQ can be used to indicate two clinically relevant issues. An RQ higher than 0.85 reliably indicates the absence of underfeeding and an RQ higher than 1.0 reliably indicates the presence of (carbohydrate) overfeeding.

\section{Acknowledgments}

The authors thank Ada van den Bos, Annelies Bos, Marjan Maliepaard, Marianne Mourik, and Ineke van Vliet for help with data collection. They also thank Ko Hagoort (Erasmus MC, Rotterdam) for careful editing.

\section{References}

[1] Biolo G, Toigo G, Ciocchi B, Situlin R, Iscra F, Gullo A, et al. Metabolic response to injury and sepsis: changes in protein metabolism. Nutrition 1997;13(suppl):52S-7.

[2] Pollack MM, Ruttimann UE, Wiley JS. Nutritional depletions in critically ill children: associations with physiologic instability and increased quantity of care. JPEN 1985;9:309-13.

[3] Coss-Bu J, Jefferson L, Walding D, David Y, Smith EO, Klish WJ. Resting energy expenditure and nitrogen balance in critically ill pediatric patients on mechanical ventilation. Nutrition 1998;14:649-52.

[4] Joosten KF, Verhoeven JJ, Hazelzet JA. Energy expenditure and substrate utilization in mechanically ventilated children. Nutrition 1999; 15:444-8.

[5] Phillips R, Ott L, Young B, Walsh J. Nutritional support and measured energy expenditure of the child and adolescent with head injury. J Neurosurg 1987;67:846-51.

[6] McClave SA, Lowen CC, Kleber MJ, Nicholson JF, Jimmerson SC, McConnell JW, et al. Are patients fed appropriately according to their caloric requirements? JPEN 1998;22:375-81.

[7] McClave SA, Lowen CC, Kleber MJ, McConnell JW, Jung LY, Goldsmith LJ. Clinical use of the respiratory quotient obtained from indirect calorimetry. JPEN 2003;27:21-6.

[8] Chwals WJ. Overfeeding the critically ill child: fact or fantasy? New Horiz 1994;2:147-55.

[9] Knauth A, Baumgart S. Accurate, noninvasive quantitation of expiratory gas leak from uncuffed infant endotracheal tubes. Pediatr Pulmonol 1990;9:55-60.

[10] Report of the Second Task Force on Blood Pressure Control in Children. Task Force on Blood Pressure Control in Children. National Heart, Lung, and Blood Institute, Bethesda, Maryland. Pediatrics 1987;79:1-25.

[11] Pollack MM, Ruttimann UE, Getson PR. Pediatric risk of mortality (PRISM) score. Crit Care Med 1988;16:1110-6.

[12] Gerver W, De Bruin R. Paediatric morphometrics: a reference manual. Utrecht: Bunge; 1996.

[13] Weir J. New methods for calculating metabolic rate with special reference to protein metabolism. J Physiol 1949;109:1-9.

[14] Joosten KF, Jacobs FI, van Klaarwater E, Baartmans MG, Hop WC, Merilainen PT, et al. Accuracy of an indirect calorimeter for mechanically ventilated infants and children: the influence of low rates of gas exchange and varying FIO2. Crit Care Med 2000;28:3014-8.

[15] McClave SA, Snider HL. Use of indirect calorimetry in clinical nutrition. Nutr Clin Pract 1992;7:207-21.

[16] Ashworth A, Millward DJ. Catch-up growth in children. Nutr Rev 1986;44:157-63. 
[17] Fjeld CR, Schoeller DA, Brown KH. Body composition of children recovering from severe protein-energy malnutrition at two rates of catch-up growth. Am J Clin Nutr 1989;50:1266-75.

[18] Joosten KF, de Kleijn ED, Westerterp M, de Hoog M, Eijck FC, Hop WCJ, et al. Endocrine and metabolic responses in children with meningococcal sepsis: striking differences between survivors and nonsurvivors. J Clin Endocrinol Metab 2000;85:3746-53.

[19] Bouwmeester NJ, Anand KJ, van Dijk M, Hop WC, Boomsma F, Tibboel D. Hormonal and metabolic stress responses after major surgery in children aged 0-3 years: a double-blind, randomized trial comparing the effects of continuous versus intermittent morphine. Br J Anaesth 2001;87:390-9.

[20] De Klerk G, Hop WC, De Hoog M, Joosten KF. Serial measurements of energy expenditure in critically ill children: useful in optimizing nutritional therapy? Intensive Care Med 2002;28:1781-5.
[21] Goran MI, Peters EJ, Herndon DN, Wolfe RR. Total energy expenditure in burned children using the doubly labeled water technique. Am J Physiol 1990;259:E576-85.

[22] Briassoulis G, Tsorva A, Zavras N, Hatzis T. Influence of an aggressive early enteral nutrition protocol on nitrogen balance in critically ill children. J Nutr Biochem 2002;13:560-9.

[23] Vo N, Waycaster M, Acuff R, Lefemine A. Effect of postoperative carbohydrate overfeeding. Ann Surg 1987;53:632-5.

[24] Bresson JL, Bader B, Rocchiccioli F, Mariotti A, Ricour C, Sachs C, et al. Protein-metabolism kinetics and energy-substrate utilization in infants fed parenteral solutions with different glucose-fat ratios. Am J Clin Nutr 1991;54:370-6.

[25] McClave S, Snider HL, Greene L, Lowen C, Cornelius M, Samuelson $\mathrm{S}$, et al. Effective utilization of indirect calorimetry during critical care. Intensive Care World 1992;9:194-200. 